MathQuiz

\section{A Game App for M-Learning}

\author{
José Marcelo Silva Santiago \\ Universidade Estadual do Ceará - \\ UECE \\ Limoeiro do Norte, Ceará \\ marcelo.gmcf@gmail.com
}

\author{
Monck Charles Nunes de \\ Albuquerque \\ Universidade Estadual do Ceará - \\ UECE \\ Fortaleza, Ceará \\ monck.albuquerque@uece.br
}

\author{
Fernanda Kécia de Almeida \\ Universidade Estadual do Ceará - \\ UECE \\ Limoeiro do Norte, Ceará \\ fernanda.kecia@uece.br
}

\author{
Francisco Ranulfo Freitas \\ Martins \\ Universidade Estadual do Ceará - \\ UECE \\ Limoeiro do Norte, Ceará \\ ranulfo.freitas@uece.br
}

\author{
Yuri Soares de Oliveira \\ Instituto Federal de Educação, Ciência \\ e Tecnologia do Ceará - IFCE \\ Fortaleza, Ceará \\ yuri.oliveira@ppgcc.ifce.edu.br
}

\begin{abstract}
This paper presents the MathQuiz application as an option to gamify some mathematics classes in elementary education II. Basically, MathQuiz is a question and answer quiz where the teacher can register questions. MathQuiz was developed for android and can be easily installed on mobile phones. MathQuiz was created in a TCC job, has been tested and perfected, and is ready to be used in classrooms.
\end{abstract}

\section{KEYWORDS}

Game, M-learning, Math

\section{INTRODUÇÃO}

O desenvolvimento de softwares alterou significativamente o modo e estilo de vida das pessoas ao redor do mundo. Desde o seu surgimento, o software se estabeleceu como a mais importante tecnologia no cenário mundial, algo inimaginável há 60 anos [16]. Nas últimas décadas, a capacidade das tecnologias Digitais da comunicação e informação (TDIC) $)^{1}$ aumentou de forma exponencial e vem revolucionando vários setores, inclusive a educação [1].

A disseminação dos softwares e a introdução a cultura digital fez com que a maioria das pessoas tivessem seus hábitos alterados [8]. Este panorama ocorre principalmente quando falamos dos nascidos digitais, uma geração de crianças e adolescentes que já nasceram num contexto de imersão ao ambiente online. Novas gerações são adeptas das funcionalidades da mídia digital, permitindo novas ações comunicativas descentradas e mobiles [5].

\footnotetext{
${ }^{1}$ Segundo [11], TDIC são tecnologias que têm o computador e a Internet como instrumentos principais e se diferenciam das Tecnologias de Informação e Comunicação (TIC) pela presença do digital.

In: XV Workshop de Trabalhos de Iniciação Científica (WTIC 2018), Salvador, Brasil. Anais do XXIV Simpósio Brasileiro de Sistemas Multimídia e Web: Workshops e Pôsteres. Porto Alegre: Sociedade Brasileira de Computação, 2018.

(c) 2018 SBC - Sociedade Brasileira de Computação.

ISBN 978-85-7669-435-9.
}

Atualmente, os dispositivos móveis, como smartphones e tablets, se tornaram itens quase indispensáveis no cotidiano das pessoas. Nesses dispositivos, um dos sistemas operacionais mais utilizados é o Android da Google[6]. Hoje na sala de aula quase todos os alunos têm um celular que pode ser utilizado para auxiliar no processo de aprendizado do aluno, portanto a educação presencial pode ser significativamente modificada com base na tecnologia, pois permite que as escolas e universidades saiam do limite geográfico para trocar informações, facilitando as pesquisas e construindo o conhecimento.

A educação, como o processo de aprender, pode ser otimizada pela possibilidade de integração de várias mídias, acessando-as tanto em tempo real como assincronicamente, assim como também pela facilidade de pôr em contato educadores e educandos [12].

Assim, como várias outras instituições e meios de comunicação estão se adequando para atender às necessidades das TDICs, a escola, enquanto espaço propício para integrações, também precisa entrar na chamada cultura da convergência, trazendo para suas práticas os benefícios da tecnologia [10].

Partindo disso, propusemos uma análise desses benefícios das TDICs para ajudar no processo de aprendizagem, olhando especificamente para as dificuldades enfrentadas na aprendizagem de Matemática nas séries do Ensino Fundamental II. A proposta foi desenvolver um jogo para celular, que o professor possa usar na sala de aula, permitindo que os alunos superem suas dificuldades e possam compreender melhor os assuntos sobre Matemática.

$\mathrm{Na}$ literatura nacional são encontrados vários trabalhos que relatam o uso de jogos na educação, porém são softwares destinados a computadores. Quando trazemos essa abordagem para o contexto de dispositivos móveis (smartphones e tablets), percebemos que ainda é tímido o número de trabalhos para essa área em específico, levando em consideração o crescimento no uso desses dispositivos móveis [15].

A metodologia utilizada nessa pesquisa, parcialmente adaptada de [7] foi focada nos alunos do Ensino Fundamental II para a experimentação de um Jogo Educativo. Foi proposto aos alunos a utilização do Jogo para responder a perguntas de matemática voltadas ao seu 
ano de ensino. Foram coletadas, por meio de questionários, algumas informações após o uso do Jogo Educativo.

Para o desenvolvimento do Jogo Educativo foi utilizado o Modelo incremental no processo de desenvolvimento de softwares, foram definidos os requisitos funcionais e não funcionais como também a relação das regras de negócios. Foram montados os diagramas de caso de uso, o diagrama de classe e seus atributos para a confecção do banco de dados.

O artigo está organizado em seis seções. A seção 1 apresenta uma introdução da utilização das tecnologias na educação e os objetivos da pesquisa. A seção 2, apresenta jogos educacionais. $\mathrm{Na}$ seção 3, alguns trabalhos relacionados com o objeto de pesquisa. $\mathrm{Na}$ seção 4, apresenta-se o Jogo Educativo MathQuiz, descrevendo sua dinâmica de funcionamento. Na seção 5 , realiza-se uma analise da usabilidade para validação do uso do Jogo. A última seção apresenta as conclusões e sugestões para trabalhos futuros.

\section{JOGOS EDUCACIONAIS}

No ambiente educacional é importante estimular o acesso a outros meios que possam dar apoio no processo de ensino e aprendizado. Segundo [9], os Jogos Educativos auxiliam na construção do conhecimento e aprendizagem.

Segundo [13], aprendizagem é vista por muitas pessoas como um "dever"para as crianças. Esta visão crítica faz com que a aprendizagem seja encarada como um trabalho. Entretanto, quando uma atividade envolve prazer, diversão, motivação, interesse e paixão, o indivíduo é capaz de dedicar a ela uma grande parte do tempo e esforço. Como jogos digitais envolvem muitos fatores motivacionais, o seu uso poderia ser estimulado por educadores para auxiliar na aprendizagem de conteúdos difíceis de tratar em salas de aula tradicionais [13].

Aprender através de ferramentas interativas não só estimula o aprendizado, como também durante um jogo o usuário se diverte e se sente desafiado para concluir as missões propostas pelo jogo, sendo uma maneira mais eficaz de ensinar $[9,14]$.

Além da facilidade em aprender através dos jogos, segundo um estudo da Federação de Cientistas Americanos [17], os jogos facilitam a interação social, pois $70 \%$ das crianças e adolescentes estudam em conjunto, resolvendo os problemas nos jogos utilizando estratégias conjuntamente[17].

\section{TRABALHOS RELACIONADOS}

Na internet, podemos encontrar diversos jogos envolvendo conteúdos de matemática, porém, poucos permitem uma personalização por parte do professor.

O jogo "O homem que calculava" proposto por [13] tem o enredo baseado no livro ${ }^{2}$. Sendo um jogo de perguntas e respostas. Foi dividido em seis estágios para se tornar mais atrativo e motivador, com níveis de dificuldade crescente. Foi desenvolvido para a plataforma Android e possui o código aberto.

Outro jogo relacionado é o Math4Mobile [4] que permite aos alunos esboçar gráficos, usando sete ícones. Podem ser usados valores para aumentar e diminui as funções que mudam a constante

${ }^{2}$ Livro clássico brasileiro traduzido para o inglês e o espanhol, do escritor Malba Tahan, pseudônimo do professor de Matemática Júlio Cesar de Mello e Souza de inclinação. O Jogo fornece feedback imediato sobre o gráfico desenhado.

Uma proposta educativa é apresentada no jogo M-learnMat [3] que é um modelo pedagógico que visa orientar atividades de $m$ learning (mobile learning) em matemática. O mesmo é embasado pela teoria da atividade e tem como foco o ensino superior. Também adota o modelo pergunta e resposta.

[18] realizou uma mapeamento esquemática sobre Jogos móveis para o aprendizado de matemática e encontrou 130 publicações. Após a aplicação de um segundo filtro, selecionou 10 artigos que melhor representa o processo de ensino e aprendizagem para matemática. Desse total apenas $25 \%$ era destinado ao ensino fundamental e $12,5 \%$ era na plataforma Android.

O diferencial entre os trabalhos apresentados nesta seção e o Jogo MathQuiz é que ele não é finito, pois este, possibilita ao professor o cadastramento de questões e imagens de acordo com o conteúdo abordado em sala de aula.

\section{SOBRE O JOGO MATHQUIZ}

Esta seção apresenta o Jogo Educativo MathQuiz e suas principais funcionalidades. A tela da (Figura 1a) é a principal após o carregamento inicial. Nela, o usuário tem a opção de escolher a série que está cursando, constituída por alternativas entre o $6^{\circ}$ ao $9^{\circ}$ ano do Ensino Fundamental II.

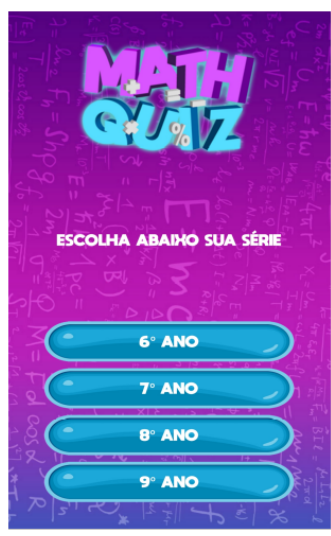

(a)

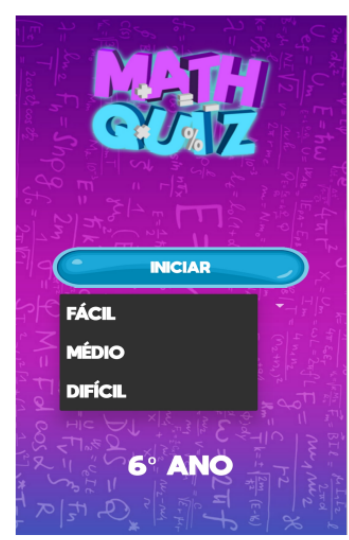

(b)
Figura 1: Telas do jogo MatQuiz. (a) Tela inicial com escolha da série (b) tela com a escolha do nível de dificuldade.

O usuário então, será direcionado para uma outra tela contendo a descrição dos níveis das perguntas presentes no Jogo Educativo (Figura 1b). O Jogo conta com três níveis de dificuldades: Fácil, Médio e Difícil.

É apresentada na (Figura 2a) a interface gráfica que o usuário vê e interage. São exibidos: o número da questão, a quantidade de pontos acertados, o cronômetro de resposta, a pergunta exibida no quadro, as opções de respostas, o botão de confirmar a opção selecionada, e o ícone azul de ajuda no canto inferior direito da tela.

$\mathrm{Na}$ (Figura 2b) é apresentada a tela em que o usuário seleciona umas das alternativas para a pergunta e o resultado encontra-se errado. Logo, é mostrada na tela uma caixa de diálogo com a mensagem "ERROU!" na cor vermelha, demonstrando que a escolha da 


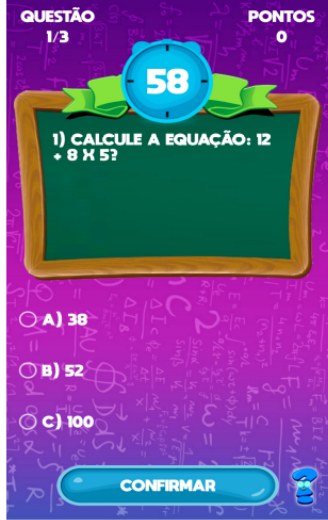

(a)

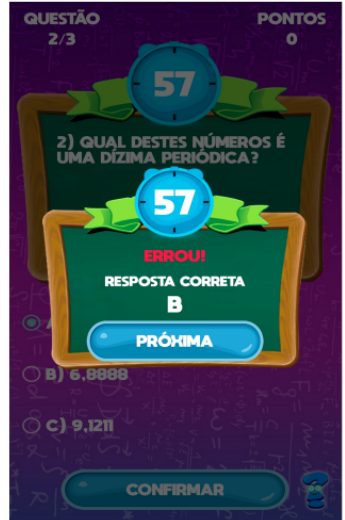

(b)
Figura 2: Telas do Jogo Educativo MatQuiz. (a) tela de exibição da questão e as opções de escolha (b) Tela sinalizando a resposta errada.

resposta não foi a correta. Em seguida, a resposta correta é mostrada na (Figura 3a).

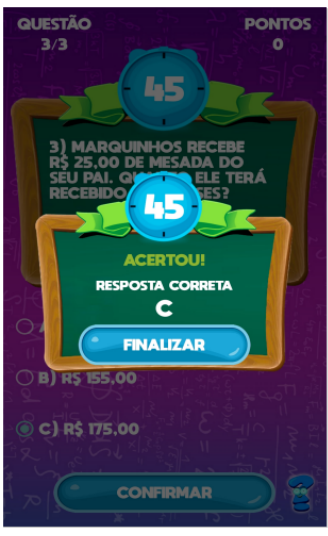

(a)

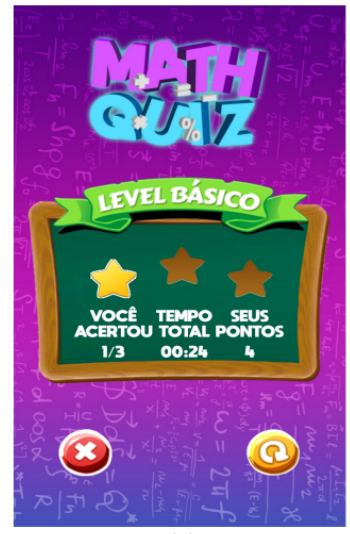

(b)
Figura 3: Telas do Jogo MatQuiz. (a) tela sinalizando o acerto da questão (b) tela de pontuação.

Por fim, a (Figura 3b) mostra o resultado final após a realização das perguntas do quiz, em que as estrelas representam a conquista em cada nível respondido com $100 \%$ de acertos nas perguntas. Abaixo, podemos ver a quantidade de acertos do usuário em cada nível, o tempo total gasto para responder no nível de dificuldade selecionado pelo usuário e a quantidade de pontos totais. No canto inferior esquerdo da tela, é exibido um ícone vermelho com um " $\mathrm{x}$ " que tem como função sair da aplicação. Já no canto inferior direito da tela há a opção de reiniciar o quiz.

\section{RESULTADOS}

O presente estudo investigou a utilização do MathQuiz na Disciplina de Matemática em duas turmas do Ensino Fundamental II. No total de 45 alunos, sendo 21 pertencentes ao $7^{\circ}$ ano e 24 do $8^{\circ}$ ano.

\subsection{Análise da Usabilidade}

Foi utilizado o Questionário de Usabilidade (SUS) para a avaliação do Jogo educativo. Para facilitar a compreensão, o grau de usabilidade está indicado no formato numérica, sendo 1 para Discordo completamente, 3 para Neutro, e 5 para Concordo completamente. A seguir, as respostas para algumas das questões contidas no questionário.

Para a frequência do uso foram obtidos os valores de $71,11 \%$ dos alunos gostariam de usar o Jogo com frequência, enquanto $11,11 \%$ não o utilizariam frequentemente (Figura 4).

\section{Eu acho que gostaria de usar esse sistema com frequência}

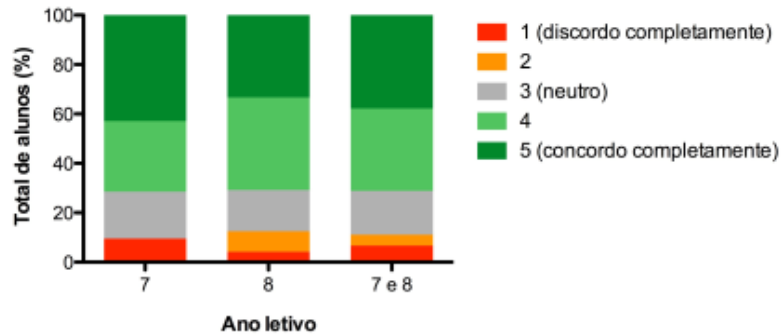

Figura 4: Frequência de Uso do Jogo.

$\mathrm{Na}$ (Figura 5), é possível notar que os usuários confirmam que o Jogo é realmente fácil de usar, pois $88,89 \%$ deles acham que outras pessoas aprenderiam rapidamente a utilizar o software.

\section{Eu imagino que as pessoas aprenderão como usar esse sistema rapidamente.}

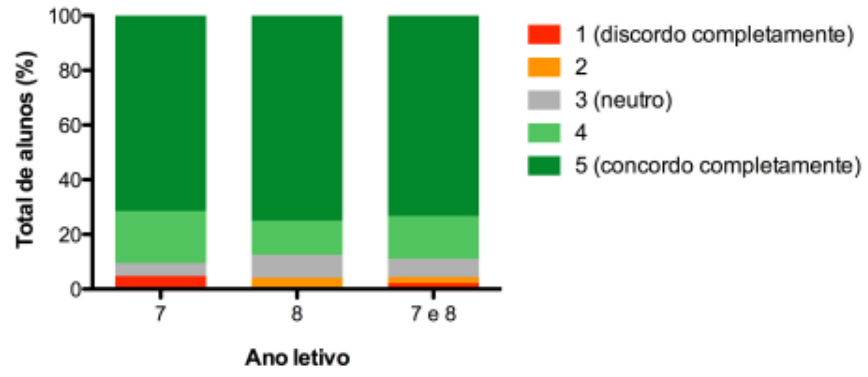

Figura 5: Facilidade de Uso do Jogo.

Para as perguntas sobre complexidade da utilização foi observado que os alunos não acharam o sistema complexo para ser utilizado, pois somente $22,22 \%$ dos estudantes apontaram que o seu uso demanda uma certa complexidade. Foi percebido que o Jogo Educativo pode ser utilizado de forma autônoma pelos estudantes. A ausência da necessidade de conhecimentos técnicos prévios foi indicado por $60 \%$ dos alunos e que os usuários confirmam que o Jogo é realmente fácil de usar, pois $88,89 \%$ deles acham que outras pessoas aprenderiam rapidamente a utilizar o software. 


\section{CONCLUSÃO}

Neste artigo foi apresentado o MathQuiz que surgiu como um trabalho de TCC da graduação em computação com o objetivo de ser um Jogo Educativo para o apoio no ensino da matemática para alunos do ensino fundamental.

A validação foi realizada com professores e alunos. O Jogo pode ser utilizado em celular ou Tablet e pode ser obtido gratuitamente.

Como trabalhos futuros para o MathQuiz foi proposto:

- Implementar modelo de competições em grupo;

- Desenvolver um Ranking da qualidade das respostas baseado em [2];

- Implementar um Cálculo de eficiência em respostas utilizando Lógica Fuzzy ${ }^{3}$;

- Realizar um estudo com uma amostra maior e heterogênea de alunos e professores.

Os resultados da pesquisa mostram indícios de que o Jogo Educativo MathQuiz é adequado para o público alvo e que é útil para apoiar o processo de ensino e aprendizagem na disciplina de matemática.

\section{REFERÊNCIAS}

[1] Monck Albuquerque, Gilvandenys Leite Sales, Pedro Rebouças Filho, and Claudio Medeiros. 2017. Avaliação da presencialidade em um fórum LV utilizando Lógica Fuzzy. In Brazilian Symposium on Computers in Education (Simpósio Brasileiro de Informática na Educação-SBIE), Vol. 28. 1357. http://dx.doi.org/10.5753/cbie.sbie. 2017.1357

[2] Leandro Amancio and Carina Friedrich Dorneles. 2017. Towards Recency Ranking in Community Question Answering: A Case Study of Stack Overflow. In Proceedings of the 23rd Brazillian Symposium on Multimedia and the Web. ACM, 173-180.

[3] Silvia Cristina F Batista, Patricia Alejandra Behar, and Liliana Maria Passerino. 2011. M-learnMat: Aplicação de um Modelo Pedagógico para Atividades de M-learning em Matemática. In Brazilian Symposium on Computers in Education (Simpósio Brasileiro de Informática na Educação-SBIE), Vol. 1.

[4] Galit Botzer and Michal Yerushalmy. 2007. Mobile application for mobile learning. In Proceedings of IADIS International Conference on Cognition and Exploratory Learning in Digital Age (CELDA 2007). 7-9.

[5] Belarmino César Guimarães da Costa. 2010. Comunicação e Educação na Era digital: reflexões sobre estética e virtualização. Revista Comunicação, Mídia e Consumo 7, 19 (2010).

[6] Emerson Barbosa da Cunha, Daniella Dias Cavalcante da Silva, and César Rocha Vasconcelos. 2017. Enabling Full Interaction with the Android System and Applications through Speech Recognition. In Proceedings of the 23rd Brazillian Symposium on Multimedia and the Web. ACM, 497-500.

[7] Dayane de Souza, Genarde Trindade, Priscila Silva Fernandes, and Bruno Bonifácio. 2017. alfabetzAR: Uma Aplicação Móvel com base na Realidade Aumentada como Ferramenta de Apoio no Processo de Alfabetização de Portadores de Síndrome de Down. In Brazilian Symposium on Computers in Education (Simpósio Brasileiro de Informática na Educação-SBIE), Vol. 28. 897.

[8] Adrián Domínguez, Joseba Saenz-De-Navarrete, Luisde-Marcos, Luis FernándezSanz, Carmen Pagés, and José-Javier Martínez-Herráiz. 2013. Gamifying learning experiences: Practical implications and outcomes. Computers and Education 63 (2013), 380-392.

[9] Neusa Nogueira Fialho. 2008. Os jogos pedagógicos como ferramentas de ensino. In Congresso nacional de educação, Vol. 6. 12298-12306.

[10] R Koster. 2004. A theroy of fun. Paraglyph Press, New York, NY, USA.

[11] Simão Pedro MARINHO and Wolney Lobato. 2008. Tecnologias digitais na educação: desafios para a pesquisa na pós-graduação em educação. Colóquio de Pesquisa em Educação 6 (2008), 1-9.

[12] José Manuel Moran. 1997. Como utilizar a Internet na educação. Ciência da informação 26, 2 (1997)

[13] José Francisco Barbosa Neto and Fernando de Souza da Fonseca. 2013. Jogos educativos em dispositivos móveis como auxílio ao ensino da matemática. RENOTE 11, 1 (2013).

\footnotetext{
${ }^{3}$ A Lógica Fuzzy apresenta uma quantidade infinita de possibilidades entre os dois extremo, diferente da Lógica Booleana que permite apenas dois valores $(0,1)$. Fo estruturada por [19] e faz parte da área de inteligencia Artificial, muito utilizado para mensurar incertezas.
}

[14] Mariana Gomide Panosso, Silvia Regina de Souza, and Verônica Bender Haydu. 2015. Características atribuídas a jogos educativos: uma interpretação AnalíticoComportamental. Revista Brasileira de Psicologia escolar e educacional 19, 2 (2015), 233-241.

[15] Francisco Ittalo Ribeiro Pessoa, Ana Liz Souto O Araujo, Wilkerson Andrade, and Dalton Guerrero. 2017. T-mind: um Aplicativo Gamificado para Estímulo ao Desenvolvimento de Habilidades do Pensamento Computacional. In Brazilian Symposium on Computers in Education (Simpósio Brasileiro de Informática na Educação-SBIE), Vol. 28. 645

[16] R. Pressman and B Maxim. 2016. Engenharia de software: uma abordagem profissional. AMGH, Porto Alegre.

[17] Gustavo Ramos, Laura Lessa, and Diana Cabral Cavalcanti. 2017. Disease Extermination: A Digital Educational Game on Vaccines, Viruses and Bacteria. In Proceedings of the 23rd Brazillian Symposium on Multimedia and the Web. ACM, 417-420.

[18] Denise Maciel Sena, Elaine Harada T de Oliveira, and Leandro SG de Carvalho. 2014. Aplicativos móveis para o aprendizado de matemática. In Brazilian Symposium on Computers in Education (Simpósio Brasileiro de Informática na Educação-SBIE), Vol. 25. 174.

[19] Lotfi A Zadeh. 1965. Fuzzy sets. Information and control 8, 3 (1965), 338-353. 